\title{
Perspectives in Meningioma Treatment
}

\author{
Athanasios K Petridis ${ }^{1 *}$, Joost Thissen ${ }^{1}$, Friedhelm Brassel ${ }^{2}$, Dan Meila ${ }^{2}$ and Martin Scholz ${ }^{1}$ \\ ${ }^{1}$ Department of Neurosurgery, Klinikum Duisburg/Wedau Kliniken, Sana Konzern, Duisburg, Germany \\ ${ }^{2}$ Department of Neuroradiology, Klinikum Duisburg/Wedau Kliniken, Sana Konzern, Duisburg, Germany
}

\begin{abstract}
The treatment of meningiomas is still at the borderline of two or even three disciplines. Surgery, radiation and interventional neuroradiology. Even when it seems confusing, reviewing the literature allows us to draw a clear line between the therapeutic modalities. There are cases where surgery is the first line treatment and others where radiation should be first line and again others where pre-operative embolization, surgery and postsurgical radiation are the treatment options of choice. In the present short review we present four cases of different meningiomas treated surgically in our department and we review the literature to provide a flow chart, indicating which treatment modality would fit to different meningiomas. Of course the flow chart is based on the present literature and cannot be followed without restrictions and reservations. Randomized multicenter studies will help to clear this field more effectively.
\end{abstract}

\section{Keywords: Meningioma; Surgery; Radiation}

\section{Introduction}

With an incidence of 1.3-7.8/100.000 cerebral meningiomas are rare tumors [1]. The only proven external risk factor to develop a meningioma is radiation exposure [2,3] with other factors being suspected, but still unproven, like electromagnetic fields, pesticides to name a few [1]. Compared to the highly malignant tumors in the brain, meningiomas count to the less malignant ones. Fortunately, in $90 \%$ of the cases they are benign lesions with a low proliferating rate and a recurrence rate of $3 \%$ in 5 years [4].

However, there are also meningiomas, which do not behave benign. These are the anaplastic meningiomas with high proliferation rates [5] and a reported recurrence rate of about $78 \%$ in five years [4] with a median recurrence time of 3.5 years [4,5]. Apart from the histology the location of a benign meningioma can be a limiting factor for a complete resection and therefore negatively affects the prognosis. Easy to remove, small $(2-4 \mathrm{~cm})$ benign meningiomas on the convexity do not cause problems for surgical removal and no further therapy is required. However, meningiomas of the petroclival area, cavernous sinus and parasagittal area are in proximity to major vessels and cranial nerves rendering their complete resection sometimes impossible and requiring further treatment with radiation or palliative embolization.

In the present short review four cases of surgery are shown, in areas were a complete resection of a meningioma (including the dura) is difficult, and the treatment modalities of meningiomas in general will be discussed.

\section{Illustrative Cases}

\section{Case 1}

Figure 1A shows a meningioma in a 77 years old female, which is adherent to the wall of the superior sagittal sinus. The meningioma is posterior to the central gyrus displacing the motoric area anteriorly. Surgery on the patient was performed and the tumor was removed completely (Figure 1B) with exception of the area which was adherent to the prominent superior sagittal sinus (Operation video 1). The patient left the hospital after 7 days in an excellent neurological condition. Histologically the meningioma was a transitional cell meningioma (World Health Organisation (WHO) grade I). Since the tumor, which was left behind was very small and the meningioma was WHO grade I, we decided to observe the patient with serial MRIs every 6 months the first year and then annually. No further treatment was recommended.

\section{Case 2}

This is the case of a 49 years old female with a transient hemihypesthesia. Diagnostic evaluation (Figure 2A) with an MRI showed a petroclival meningioma with compression of the pons and of cranial nerves IV, V and VII-VIII complex. Surgery was performed and the tumor was removed completely although the dura was not removed but coagulated (Operation video 2). Postsurgical cranial CT does not show any signs of bleeding or residual tumor (Figure 2B). Histology showed a meningothelial meningeoma (WHO grade I). Because of the benign nature of this meningioma we decided to observe the patient in 6 months intervals since microscopically the possibility of having left tumor cells behind was significant.

\section{Case 3}

The angiography shows a highly vascularized tumor, which was preoperatively embolized. After embolization the tumor was completely excluded from its vascular supply (Figure 3A and 3B) and could be removed without problems. The meningioma was vascularized almost exclusively by dural feeders and superselective embolization of the meningioma feeders through the media meningeal artery was possible and led to a complete disappearance of the tumor: Follow up MRI two years after embolization.

\section{Case 4}

This is the case of a 58 years old male with a highly vascularized meningioma (Figure 4A), which was not embolized. During surgery, bleeding was significant and made the surgical removal of the tumor very challenging (operating video 3 ). Immediately after tumor removal and coagulation of the feeders the bleeding stopped. Postsurgical cranial CT shows a complete tumor removal with no residual bleeding (Figure $4 \mathrm{~B})$. The patient left the hospital after 7 days without neurological

*Corresponding author: Athanasios K. Petridis, Department of Neurosurgery Sana Konzern, Wedau Kliniken Duisburg, Zu den Rehwiesen 9, 45077 Duisburg, Germany, Tel: +49-203-7332425; E-mail: opticdisc@aol.com

Received December 16, 2014; Accepted February 06, 2015; Published February 08, 2015

Citation: Petridis AK, Thissen J, Brassel F, Meila D, Scholz M (2015) Perspectives in Meningioma Treatment. J Neurol Disord 3: 223. doi:10.4172/2329-6895.1000223

Copyright: (c) 2015 Petridis AK, et al. This is an open-access article distributed under the terms of the Creative Commons Attribution License, which permits unrestricted use, distribution, and reproduction in any medium, provided the original author and source are credited. 
Citation: Petridis AK, Thissen J, Brassel F, Meila D, Scholz M (2015) Perspectives in Meningioma Treatment. J Neurol Disord 3: 223. doi: 10.4172/2329-6895.1000223

Page 2 of 4

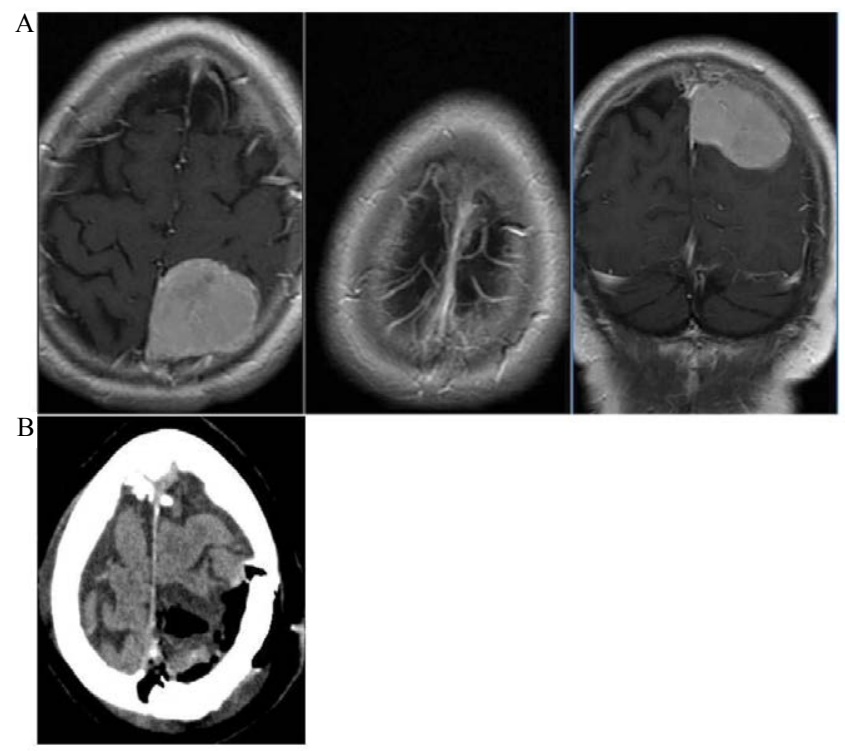

Figure 1: (A) 77 year old female with a parasagital meningioma. The size and superficial location as well as the proximity to the motoric area exclude first line irradiation. Surgery was performed. The tumor is shown in this contrast enhanced MRI (T1W). Contrast enhancement is homogenous. Left and middle images. axial section, right image: coronal section. (B) Post-surgical cranial CT shows no residual tumor although during surgery a thin layer of tumor on the lateral sagital sinus wall was left to protect lesioning the strong sinus.
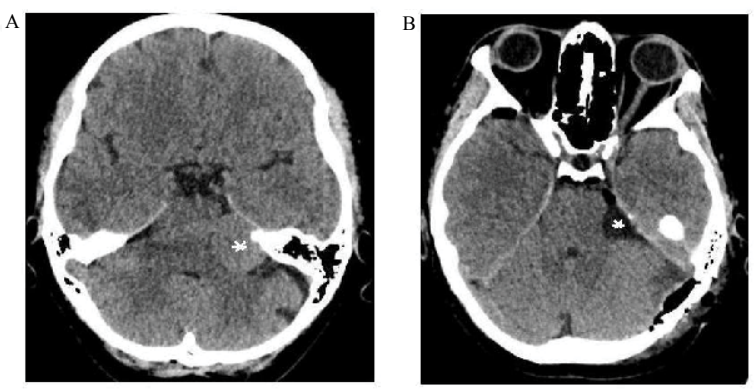

Figure 2: (A) 49 year old female with a meningioma of the petroclival area without invasion into the middle fossa. Surgery was performed and gross total resection without lesioning the cranial nerves had been performed. (B) Postsurgical cranial CT shows the complete tumor removal. The asterisk indicates the tumor.

deficits. Histology showed a meningothelial meningioma WHO grade I. Observation in a 6 months interval the first year and afterwards annually for 5 years with MRI was recommended.

\section{Discussion}

Even when generally benign tumors, meningiomas can prove themselves as very challenging to treat. Depending on histology, vascularity and localization a multimodal treatment with angiographical embolisation pre-surgically, surgery and postsurgical irradiation can be required. The toughest enemy is the one, which is underestimated.

Anaplastic meningiomas are only 1-3\% of meningioma cases but have a high proliferation rate and behave very malignant with a five year survival of about 50\% [6,7]. These tumors cannot be considered treated by surgical removal alone. At the moment the major obstacle in the treatment of anaplastic meningiomas is the lack of standard therapeutic regimens. As discussed in a review by Rogers [8] prospective trials assessing the role of radiation and or surgery with standardization of response criteria as well as outcome end points do not exist, but are about to come (Phase II and III trials of the Radiation Therapy Oncology Group and the European Organization for Research and Treatment of Cancer). New diagnostic methods like PET imaging with application of somatostatin receptor ligands in skull base meningiomas will prove themselves of additional help in further delineation of meningiomas [9].

Fractionated stereotactic radiotherapy with and without surgery seems also to be an effective tool in the armamentarium of meningiomas treatment as shown in meningioma grade I [10] allowing meningiomas which are unresectable or only resectable with great risk (cavernous sinus) to be effectively treated. With special focus on the dural tail, which is left in some cases of cerebellopontine and parasagittal meningiomas there is a study which indicated that gamma knife radiosurgery of meningiomas with exclusion of the dural tail did not lead to out-offield tumor progression although the median follow up time was 41 months [11]. When tumor progression was seen it was always in the radiation target area and never in the dural tail (MRI enhancing dura bordering the tumor). This conclusion encourages individual decisions to coagulate the dura and leave the dural tail in cases where removal could endanger the patient. On the other hand histological analysis of the dural tail showed that in 22 of 36 cases tumor cells were seen [12]. Additionally, this study shows that gamma knife is highly effective in treating grade I meningiomas with 2- and 5- year control rates of $95 \%$ and $88 \%$ respectively. However, the local control rate of WHO grade II meningiomas was $83 \%$ and $64 \%$ after 2 - and 5 years.

In petroclival meningiomas the gross total resection can be very challenging leading to serious neurological deficits. Little et al. [13] showed that even the near total resection of such meningiomas did not lead to a significantly increased rate of tumor recurrence. The authors performed a near total resection in adherent and fibrous tumors, which is in accordance to our regimen. Hashemi et al. [14] concluded that removal of tentorial fold meningiomas can lead to a high rate of
A

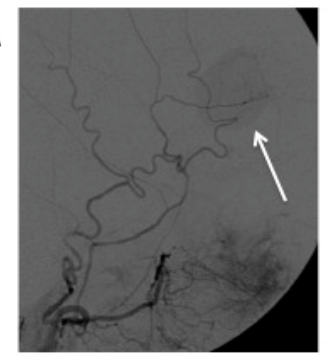

C

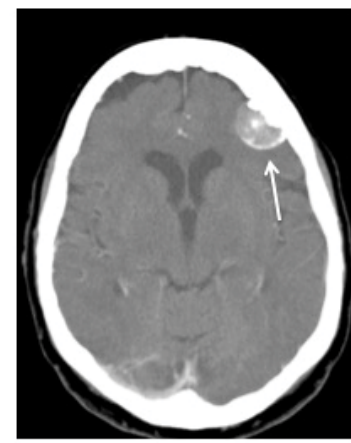

$B$

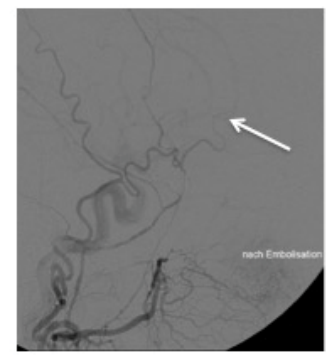

D

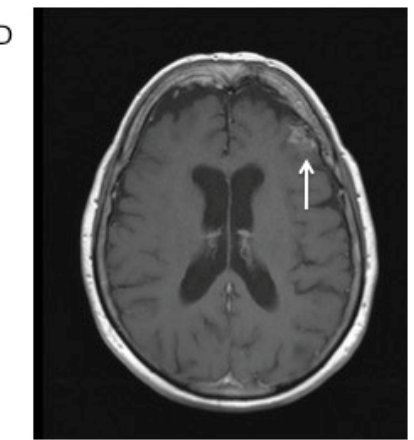

Figure 3: (A) Angiography of a highly vascularized meningioma (arrow). (B) Post-embolisation. The tumor seems to be completely devascularized. (C) $\mathrm{CCt}$ before embolization (arrow indicates the tumor). (D) MRI 2 years after embolisation of the meningioma showing a regression of the tumor growth. 


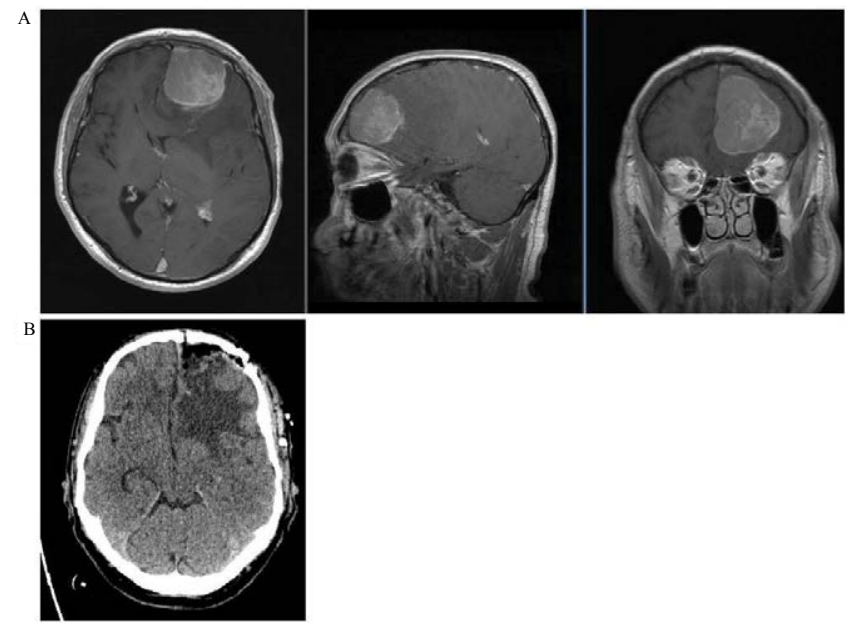

Figure 4: (A) 58 year old male with a meningioma. Hypervascularisation is not seen and therefore no embolisation had been performed. The tumor enhances homogenously in the T1W contrast enhanced MRI in all three planes, axial, sagittal and coronal. (B) Post-surgical cranial CT shows no bleeding and no residual tumor.

neurological morbidity and recommend the volume reductive surgery in tentorial meningiomas when extend into the anterior portion of the middle fossa is present, followed by radiation therapy.

Radiation therapy of cavernous sinus meningiomas showed a progression free survival rate of $93 \%$ at a 10 year follow up, encouraging radiation therapy as first or second line treatment in cases were surgery cannot be performed [15].

In highly vascularized meningiomas, embolisation should be considered before surgery, since devascularisation induces necrosis and facilitates resection, reduces blood loss and operating time and increases the possibility to completely remove the tumor [16]. In contrast to the gain of angiographic embolisation there are also some risk factors of the method, like tumor hemorrhage or ischemia from an untargeted vessel embolisation, ischemia of cranial nerves, edema with mass effect and seizures to name a few [16]. Meningiomas, which are vascularized by dural feeders are a good target for superselective embolization with an excellent therapeutic or palliative outcome and can be very helpful in the preoperative treatment. Meningiomas with feeders from leptomeningeal vessels on the other hand should not be treated by embolization.

Last but not least there is another complication of meningioma surgery, which has to be considered in the therapeutic plan. The postoperative thromboembolic complication risk after meningioma surgery, which is shown to have a 90 -day mortality rate of $11 \%$, depending on risk factors like bedridden and adipose patients [17]. In patients with such risk factors and older age, radiation or even in the lack of symptoms, observation can be considered.

The general strategy in meningioma surgery is the following: 1) atraumatic approach and opening possible cisterns to gain cerebrospinal fluid, 2) coagulation of the tumor feeders in order to stop blood supply of the tumor, 3) debulking of the tumor and 4) preparation of the tumor border. It is very important to hold tight to this systematic rule in all cases because too early debulking process can cause major bleeding (see case 4). In convexity meningiomas the blood supply to the tumor can be easily stoped, only by coagulation of the dura before opening the dura itself. In complicated tumors like pertroclival tumors the neurosurgeon has to coagulate between the different origins of the cranial nerves more in a segmented manner. Although the best strategy is not to use coagulation close to cranial nerves at all. The debulking process is mainly influenced by the consistency of the tumor. If the tumor is hard, one has to cut out tumor pieces, if it is soft the tumor can be reduced exclusively with the suction tube. The ultrasonic aspirator (CUSA) can be very helpful.

Especially close to the optic nerve, menigioma surgery is the better alternative since radiation close to the optic nerve is not without higher risks.

\section{Conclusion}

In conclusion the present data in the literature show similar good results for surgery and radiation therapy in meningioma grade I. Meningiomas that are surgically approachable without high risk of neurological morbidity should be treated by surgical removal to spare the patients of postradiation complications. Alternatively proton beam radiotherapy shows similar good results compared to high voltage photon therapy with less radiation risks [18,19]. Surgically approachable tumors are meningiomas located at the convexity. The more superficial a meningioma is, the less can the tumor be controlled by radiation therapy. Additionally, to be ideal for radiotherapy, the tumor size should be less than $3-4 \mathrm{~cm}$ with a distinct margin and almost no surrounding edema, as well as with sufficient distance from eloquent tissue. Tumors which are adherent to the sagittal sinus or cranial nerves (cavernous sinus, petroclival area) should receive a near total resection followed by radiotherapy or even radiation therapy alone (size $<3-4$ $\mathrm{cm}$ ), since radiotherapy shows a satisfying outcome [20,21]. However, the risk of radiotherapy to induce a permanent neurological deficit is given with $6.3 \%$ for tumors near the cavernous sinus and the radiation induced risk of stereotactic irradiation is up to $40 \%$. These data have also to be kept in mind when a treatment decision for a meningioma is taken [21-23]. Tumors with exclusive dural feeders can be treated or pre-treated by embolization. An experienced interventionalist should be chosen for this treatment, since vessels feeding cranial nerves can be embolized too and cause a cranial nerve palsy. The conclusions of our review are illustrated in the flow chart of Figure 5.

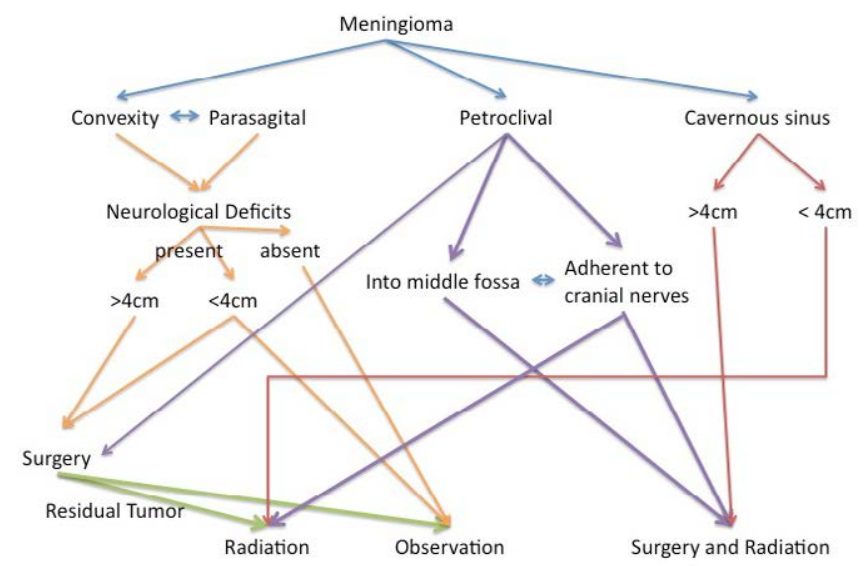

Figure 5: Flow chart to illustrate the decision algorithm in the treatment of meningiomas. The Flow charts takes into account the different most important factors for the decision tree towards surgery, observation, radiation or the combination of surgery and radiation. Tumor location, neurological deficits, size, adherence to cranial nerves are all parameters considered in the treatment strategy. A convexity meningioma with neurological deficits in example is a clear indication for surgery whereas a convexity meningioma with no neurological deficits could be observed. Typical these meningiomas (when they have no neurological deficits) are not large. Meningiomas into the cavernous sinus should preferably treated by radiation or radiation and partially surgical resection. 
Recommendations about how to treat a meningioma are not evidence based because of lack of randomized prospective multicenter studies. According to a study from Marcus et al, [24] neurosurgeons would not recommend radiotherapy in grade I meningiomas. We recommend radiotherapy in cases of meningiomas, which render a high risk of neurological morbidity at least after tumor reductive therapy. In anaplastic meningiomas radiation therapy follows surgery in most cases irrespective of complete resection [6]. Conformal high dose radiotherapy resulted in improved rates of survival in anaplastic meningiomas especially in proton beam radiation therapy [25]. Chemotherapy on the other side has been applied in some cases but did not change the prognosis favorably [26].

However, should chemotherapy become feasible, a superselective chemotherapy through endovascular approaches in tumors feeded centrifugally through dural vessels can be an excellent treatment modality.

\section{References}

1. Baldi I, Engelhardt J, Bonnet C, Bauchet L, Berteaud E, et al. (2014) Epidemiology of meningiomas. Neurochirurgie.

2. Jager B, Schuhmann MU, Schober R, Kortmann RD, Meixensberger J (2008) Induction of gliosarcoma and atypical meningioma 13 years after radiotherapy of residual pilocytic astrocytoma in childhood. Pediatr Neurosurg 44: 153-158.

3. Reynier Y, Guillermain P, Jazzar A, Vigouroux RP (1986) [Radiation-induced meningioma. 3 cases]. Neurochirurgie 32: 135-139.

4. Jääskeläinen J, Haltia M, Servo A (1986) Atypical and anaplastic meningiomas: radiology, surgery, radiotherapy, and outcome. Surg Neurol 25: 233-242.

5. Petridis AK, Doukas A, Mahvash M, Dörner L, Hugo HH, et al. (2009) A case of rapid-growing anaplastic meningiomas. BMJ Case Rep 2009.

6. Ahmeti $\mathrm{H}$, Maslehaty H, Petridis AK, Doukas A, Mahvash M, et al. (2013) Extensive growth of an anaplastic meningioma. Case Rep Neurol Med 2013: 527184.

7. Mahmood A, Caccamo DV, Tomecek FJ, Malik GM (1993) Atypical and malignant meningiomas: a clinicopathological review. Neurosurgery 33: 955-963.

8. Rogers L, Barani I, Chamberlain M, Kaley TJ, McDermott M, et al. (2015) Meningiomas: knowledge base, treatment outcomes, and uncertainties. A RANO review. J Neurosurg 122: 4-23.

9. Suchorska B, Tonn JC, Jansen N (2014) PET imaging for brain tumor diagnostics. Curr Opin Neurol 27: 683-688.

10. Kaul D, Budach V, Misch M, Wiener E, Exner S, et al. (2014) Meningioma of the skull base: long-term outcome after image-guided stereotactic radiotherapy. Cancer Radiother 18: 730-735.

11. Bulthuis VJ, Hanssens PEJ, Lie ST, van Overbeeke JJ (2014) Gamma knife radiosurgery for intracranial meningiomas: Do we need to treat the dural tail? A single-center retrospective analysis and an overview of the literature. Surg Neurol Int 5: S391-S395.

12. Wen M, Jung S, Moon KS, Pei J, Lee KH, et al. (2014) Immunohistochemical profile of the dural tail in intracranial meningiomas. Acta Neurochir (Wien) 156: 2263-2273.
13. Little KM, Friedman AH, Sampson JH, Wanibuchi M, Fukushima T (2005) Surgical management of petroclival meningiomas: defining resection goals based on risk of neurological morbidity and tumor recurrence rates in 37 patients. Neurosurgery 56: 546-559.

14. Hashemi M, Schick U, Hassler W, Hefti M (2010) Tentorial meningiomas with special aspect to the tentorial fold: management, surgical technique, and outcome. Acta Neurochir (Wien) 152: 827-834.

15. Dufour H, Muracciole X, Metellus $P$, Regis J, Chinot O, et al. (2001) Longterm tumor control and functional outcome in patients with cavernous sinus meningiomas treated by radiotherapy with or without previous surgery: is there an alternative to aggressive tumor removal? Neurosurgery 48: 285-294.

16. Shah AH, Patel N, Raper DM, Bregy A, Ashour R, et al. (2013) The role of preoperative embolization for intracranial meningiomas. J Neurosurg 119: 364372.

17. Hoefnagel D, Kwee LE, van Putten EH, Kros JM, Dirven CM, et al. (2014) The incidence of postoperative thromboembolic complications following surgical resection of intracranial meningioma. A retrospective study of a large single center patient cohort. Clin Neurol Neurosurg 123: 150-154,

18. Wenkel E, Thornton AF, Finkelstein D, Adams J, Lyons S, et al. (2000) Benign meningeoma: partially resecte, biopsied. And recurrent intracranial tumors treated with combined proton and photon radiotherapy. Int J Radiat Oncol Biol Phys48: 1363-1370.

19. Combs SE, Ganswindt U, Foote RL, Kondziolka D, Tonn JC (2012) State-ofthe-art treatment alternatives for base of skull meningiomas: complementing and controversial indications for neurosurgery, stereotactic and robotic based radiosurgery or modern fractionated radiation techniques. Radiation Oncology 7: 226.

20. Alexiou GA, Gogou P, Markoula S, Kyritsis AP (2010) Management of meningiomas. Clin Neurol Neurosurg 112: 177-182.

21. Starke RM, Williams BJ, Hiles C, Nguyen JH, Elsharkawy MY, et al. (2012) Gamma knife surgery for skull base meningiomas. J Neurosurg 116: 588-597.

22. Amichetti M, Amelio D, Minniti G (2012) Radiosurgery with photons or protons for benign and malignant tumours of the skull base: a review. Radiat Oncol 7: 210 .

23. Kondziolka D, Mathieu D, Lunsford LD, Martin JJ, Madhok R, et al. (2008) Radiosurgery as definitive management of intracranial meningiomas. Neurosurgery 62: 53-58.

24. Marcus HJ Price SJ, Wilby M Santarius T, Kirollos RW (2008) Radiotherapy as an adjuvant in the management of intracranial meningiomas: are we practising evidence-based medicine? Br J Neurosurg 22: 520-528.

25. Hug EB, Devries A, Thornton AF, Munzenride JE, Pardo FS, et al. (2000) Management of atypical and malignant meningiomas: role of high-dose, 3D-conformal radiation therapy. J Neurooncol 48: 151-160.

26. Chamberlain MC (2004) Intracerebral Meningiomas. Curr Treat Options Neurol 6: 297-305 